\title{
EXACT BOUNDARY CONDITIONS AT AN ARTIFICIAL BOUNDARY FOR PARTIAL DIFFERENTIAL EQUATIONS IN CYLINDERS*
}

\author{
THOMAS HAGSTROM ${ }^{\dagger}$ AND H. B. KELLER ${ }^{\ddagger}$
}

\begin{abstract}
The numerical solution of partial differential equations in unbounded domains requires a finite computational domain. Often one obtains a finite domain by introducing an artificial boundary and imposing boundary conditions there. This paper derives exact boundary conditions at an artificial boundary for partial differential equations in cylinders. An abstract theory is developed to analyze the general linear problem. Solvability requirements and estimates of the solution of the resulting finite problem are obtained by use of the notions of exponential and ordinary dichotomies. Useful representations of the boundary conditions are derived using separation of variables for problems with constant tails. The constant tail results are extended to problems whose coefficients obtain limits at infinity by use of an abstract perturbation theory. The perturbation theory approach is also applied to a class of nonlinear problems. General asymptotic formulas for the boundary conditions are derived and displayed in detail.
\end{abstract}

AMS(MOS) subject classifications. Primary 35A05, 35A40, 35C20, 65N99

Key words. artificial boundary conditions, asymptotic expansions for PDE's

1. Introduction. Many of the boundary value problems arising in applied mathematics are given on unbounded domains. Examples include the problems of fluid flow and wave propagation in channels or past bodies. The numerical solution of these problems, however, requires a finite domain. In this paper, we develop a theory for the exact reduction of a boundary value problem for a partial differential equation on an unbounded cylindrical domain to a problem on a bounded domain. That is, an "artifical" boundary is introduced and the proper boundary condition to be imposed there is derived. In other works, [8] and [9], we use our theory to solve nonlinear problems of both elliptic and parabolic type.

For ordinary differential equations, exact reduction theories have been developed by many authors: de Hoog and Weiss [5], Keller and Lentini [11], Jepson and Keller [10] and Markowich [12]. Few works on artificial boundary conditions for partial differential equations, on the other hand, have discussed exact conditions. An exception is the paper of Gustafsson and Kreiss [6], where the form of the proper conditions for a general hyperbolic problem is derived. They go on to find representations of the exact conditions in various simple cases for problems of both hyperbolic and elliptic type.

We illustrate the derivation of exact conditions with the following example:

a) $\nabla^{2} u+a(x, \mathbf{y}) u=f(x, \mathbf{y}), \quad(x, \mathbf{y}) \in[0, \infty) \times \Omega, \quad \Omega \subset R^{n-1}$,

b) $\quad c(x, \mathbf{y}) \frac{\partial u}{\partial \nu}(x, \mathbf{y})+d(x, \mathbf{y}) u(x, \mathbf{y})=\gamma_{\Omega}(x, \mathbf{y}), \quad \mathbf{y} \in \partial \Omega$,

c) $\quad \alpha(\mathbf{y}) \frac{\partial u}{\partial x}(0, \mathbf{y})+b(\mathbf{y}) u(0, \mathbf{y})=\gamma_{0}(\mathbf{y}), \quad \mathbf{y} \in \Omega$,

d) $\lim _{x \rightarrow \infty} u(x, \mathbf{y})=0$,

e) $\quad c(x, \mathbf{y})=c_{\infty}(\mathbf{y}), \quad d(x, \mathbf{y})=d_{\infty}(\mathbf{y}), \quad a(x, \mathbf{y})=a_{\infty}(\mathbf{y}), \quad x \geqq x_{0}$, $f(x, \mathbf{y})=\gamma_{\Omega}(x, \mathbf{y})=0, \quad x \geqq x_{0}$.

${ }^{*}$ Received by the editors April 5, 1984. This research was sponsored by the U. S. Army under contract DAAG29-80-C-0041, and supported in part by the U. S. Department of Energy under contract DE-AS03$76 \mathrm{SF}-00767$.

${ }^{\dagger}$ Department of Applied Mathematics and Statistics, State University of New York, Stony Brook, New York 11794.

‡Applied Mathematics, California Institute of Technology, Pasadena, California 91125. 
(We say that (1.1) has a constant tail, due to condition (1.1e).) We choose some point $x=\tau \geqq x_{0}$ as the location of the artificial boundary. In the "tail", $x \geqq \tau$, we have:

a) $\quad \nabla^{2} u+a_{\infty}(\mathbf{y}) u=0, \quad(x, \mathbf{y}) \in[\tau, \infty) \times \Omega ;$

b) $\quad c_{\infty}(\mathbf{y}) \frac{\partial u}{\partial v}(x, \mathbf{y})+d_{\infty}(\mathbf{y}) u(x, \mathbf{y})=0, \quad \mathbf{y} \in \partial \Omega$

c) $\quad \lim _{x \rightarrow \infty} u(x, \mathbf{y})=0$.

Problem (1.2) can be easily analyzed by separation of variables. Consider the following eigenvalue problem:

a) $\quad \nabla_{\mathbf{y}}^{2} Y_{n}(\mathbf{y})+a_{\infty}(\mathbf{y}) Y_{n}(\mathbf{y})=\omega_{n} Y_{n}(\mathbf{y}), \quad \mathbf{y} \in \Omega$,

b) $\quad c_{\infty}(\mathbf{y}) \frac{\partial}{\partial \nu} Y_{n}(\mathbf{y})+d_{\infty}(\mathbf{y}) Y_{n}(\mathbf{y})=0, \quad \mathbf{y} \in \partial \Omega$;

c) $\quad \int_{\Omega} d \mathbf{y} Y_{n}^{2}(\mathbf{y})=1$.

Given certain assumptions on the boundary condition, (1.3b), the set of eigenfunctions, $\left\{Y_{n}\right\}$, is complete in that subspace of $L_{2}(\Omega)$ consisting of functions satisfying it. (See Berezanskii [3].) For simplicity, we further assume that the $\omega_{n}$ are distinct and that $\omega_{n}=0$ is not an eigenvalue. We rewrite the $\left\{\omega_{n}\right\}$ in the following way:

$$
\begin{array}{ll}
\omega_{n}=\alpha_{n}^{2}>0, & n=1, \cdots, m, \\
\omega_{n}=-\lambda_{n}^{2}<0, & n=m+1, m+2, \cdots .
\end{array}
$$

Expanding $u$ in terms of the $Y_{n}$ 's,

$$
u(x, \mathbf{y})=\sum_{n=1}^{\infty} c_{n}(x) Y_{n}(\mathbf{y})
$$

problem (1.2) becomes:
a) $c_{n}^{\prime \prime}=\left\{\begin{array}{l}-\alpha_{n}^{2} c_{n}, \\ \lambda_{n}^{2} c_{n},\end{array}\right.$
$n=1, \cdots, m$,
$n=m+1, m+2, \cdots$, $x \in[\tau, \infty)$
b) $\quad \lim _{x \rightarrow \infty} c_{n}(x)=0, \quad n=1,2, \cdots$.

As (1.6a) can be trivially solved, we see that (1.6b) is satisfied if and only if:
a) $\quad c_{n}(\tau)=c_{n}^{\prime}(\tau)=0, \quad n=1, \cdots, m$;
b) $\quad c_{n}^{\prime}(\tau)=-\lambda_{n} c_{n}(\tau), \quad n=m+1, m+2, \cdots$.

This allows us to replace (1.1) by an equivalent finite domain problem:
a) $\quad \nabla^{2} u+a(x, \mathbf{y}) u=f(x, \mathbf{y}), \quad(x, \mathbf{y}) \in[0, \tau] \times \Omega$;
b) $\quad c(x, \mathbf{y}) \frac{\partial u}{\partial \nu}(x, \mathbf{y})+d(x, \mathbf{y}) u(x, \mathbf{y})=\gamma_{\Omega}(x, \mathbf{y}), \quad \mathbf{y} \in \partial \Omega$;
c) $\quad \alpha(\mathbf{y}) \frac{\partial u}{\partial x}(0, \mathbf{y})+b(\mathbf{y}) u(0, \mathbf{y})=\gamma_{0}(\mathbf{y}), \quad \mathbf{y} \in \Omega$;
d) $\quad \int_{\Omega} d \mathbf{y} u(\tau, \mathbf{y}) Y_{n}(\mathbf{y})=\int_{\Omega} d \mathbf{y} \frac{\partial u}{\partial x}(\tau, \mathbf{y}) Y_{n}(\mathbf{y})=0, \quad n=1, \cdots, m ;$
$\int_{\Omega} d \mathbf{y} \frac{\partial u}{\partial x}(\tau, \mathbf{y}) Y_{n}(\mathbf{y})=-\lambda_{n} \int_{\Omega} d \mathbf{y} u(\tau, \mathbf{y}) Y_{n}(\mathbf{y}), \quad n=m+1, m+2, \cdots$ 
That is, (1.1) has a solution if and only if (1.8) does and the solutions agree on a finite domain.

In $\$ 2$ of this work we derive boundary conditions for the reduction of a general partial differential equation in a semi-infinite cylindrical domain to a finite one. These turn out to be the requirement that the appropriate data at the artificial boundary lie in a certain affine set. We find it convenient to rewrite the problem as an ordinary differential equation in a Banach space, making transparent the connection between our reduction and the reduction theorems for the case of ordinary differential equations. In $\$ 3$ we introduce the notion of a dichotomy for our abstract equation and use it to develop error estimates and solvability requirements for the finite problem.

We first consider the problem of representing the boundary conditions in $\S 4$. Here separation of variables is used to analyze constant tail problems such as the one presented above. The exact representation we obtain is equivalent to (1.8d) in that case.

We develop a perturbation theory to analyze nonconstant tail problems in $\$ 5$. Assuming the limiting problem at infinity can be solved by separation of variables, a perturbation expansion of the exact boundary condition can be calculated. We carry out this expansion for the Helmholtz equation exterior to a body, recovering the conditions of Bayliss, Gunzburger and Turkel [2]. Finally, in $§ 6$, nonlinear problems are considered. We use the perturbation theory of the preceding section to prove, under certain conditions, the existence of an exact nonlinear boundary condition and to calculate an expansion which approximates it.

We note that many authors have derived boundary conditions for specific problems. We do not, in general, attempt to examine the connection between their conditions and ours. For more discussion of these connections as well as for a more extensive bibliography, the reader is referred to Hagstrom [7].

2. Basic linear reduction theorem. We consider abstract boundary value problems of the form:

$$
\begin{array}{ll}
\text { a) } & \frac{d u}{d x}=A(x) u+f(x), \quad 0<x<\infty ; \\
\text { b) } & B_{0} u(0)=\gamma_{0}, \\
\text { c) } & \lim _{x \rightarrow \infty} B_{\infty} u(x)=0 .
\end{array}
$$

In addition we may impose:

d) $\quad\|u(x)\|$ bounded as $x \rightarrow \infty$.

For some Banach space, $\mathscr{B}$, we seek $u(x) \in \mathscr{B}$ for $x \in[0, \infty]$. We suppose that $A(x), B_{0}$ and $B_{\infty}$ are linear operators with domain in $\mathscr{B}$, to which we also constrain the range of $A(x)$. Finally, $f(x) \in \mathscr{B}$.

Problems of form (1.1) can be obtained from general partial differential equation problems in cylindrical domains. Specifically we consider

$$
\left(\sum_{j=1}^{n} P_{j}\left(\mathbf{y}, \mathbf{x}, \frac{\partial}{\partial \mathbf{y}}\right) \frac{\partial^{j}}{\partial x^{j}}\right) \omega=g(x, \mathbf{y})
$$

on the cylindrical domain

$$
(x, \mathbf{y}) \in[0, \infty) \times \Omega, \quad \Omega \subset \mathbf{R}^{n} .
$$


Homogeneous boundary conditions are imposed on $\partial \Omega$ involving $\omega$ and its normal derivatives;

$$
\sum_{j=0}^{m} B_{\Omega, h j}(\mathbf{y}) \frac{\partial^{j} \omega}{\partial \nu^{j}}(x, \mathbf{y})=0, \quad \mathbf{y} \in \partial \Omega .
$$

We further suppose that, subject to these boundary conditions,

$$
P_{n}^{-1}\left(x, \mathbf{y}, \frac{\partial}{\partial \mathbf{y}}\right)
$$

exists for all $x$. Now (2.2) can be rewritten in the form of (2.1a) by introducing

$$
u=\left(\begin{array}{c}
\frac{\partial^{n-1} \omega}{\partial x^{n-1}} \\
\frac{\partial^{n-2} \omega}{\partial x^{n-2}} \\
\vdots \\
\omega
\end{array}\right) .
$$

The space, $\mathscr{B}$, is some space of $n$-tuples of functions on $\Omega$ which satisfy the homogeneous boundary conditions, (2.3). It is necessary to eliminate inhomogeneous conditions on $\partial \Omega$ in order to reduce the problem to the abstract form. This can be accomplished by subtracting a function that satisfies the inhomogeneous condition. We note that the functions $B_{\Omega, j}$ affect $P_{n}^{-1}$ and, ultimately, $A(x)$.

Returning to (2.1) we choose some finite point, $x=\tau$, and attempt to reduce the infinite problem on $[0, \infty)$ to a finite one on $[0, \tau]$. We define $\mathbf{A}(\tau ; f)$, the admissible set of Cauchy data at $x=\tau$, as the set leading to solutions, $u$, in the tail, $x \in[\tau, \infty)$. More precisely we have:

Definition 2.5. The set $\mathbf{A}(\tau ; f) \subset \mathscr{B}$, the admissible set at $x=\tau$, is the set of all $u_{0} \in \mathscr{B}$ such that there exists $u(x) \in \mathscr{B}, x \in[\tau, \infty)$, satisfying:

$$
\begin{aligned}
& \text { a) } \frac{d u}{d x}=A(x) u+f(x), \quad \tau<x<\infty, \\
& \text { b) } u(\tau)=u_{0} ;
\end{aligned}
$$

as well as (2.1c, d) as appropriate.

It is now possible to write down an exact reduction of (2.1) to a problem on a finite domain. We state the reduction as a theorem whose proof follows immediately from the definition of $\mathbf{A}(\tau ; f)$. solution:

THEOREM 2.6. Problem (2.1) has a solution if and only if the following problem has a

$$
\begin{array}{ll}
\text { a) } & \frac{d \omega}{d x}=A(x) \omega+f(x), \quad x \in[0, \tau] \\
\text { b) } & B_{0} \omega(0)=\gamma_{0} \\
\text { c) } & \omega(\tau) \in \mathbf{A}(\tau ; f) .
\end{array}
$$

Furthermore, whenever (2.1) has a solution $u(x),(2.6)$ has a solution which is identical to $u$ on $[0, \tau]$. 
Proof. Suppose (2.6) has a solution. Then, by the definition of $\mathbf{A}(\tau ; f)$, there exists $u^{+}(x), x \in[\tau, \infty)$, satisfying (2.1a) and (2.1c, d) as appropriate as well as $\omega(\tau)=u^{+}(\tau)$. Define

$$
u(x)= \begin{cases}\omega(x), & x \in[0, \tau], \\ u^{+}(x), & x \in[\tau, \infty) .\end{cases}
$$

Then, $u$ is a solution of (2.1). Now, suppose that (2.1) has a solution. The restriction of $u$ to $[\tau, \infty)$ satisfies (2.5) and (2.1c, d) and, hence, by the definition of $\mathbf{A}(\tau ; f), u(\tau) \in$ $\mathbf{A}(\tau ; f)$. This implies that the restriction of $u$ to $[0, \tau]$ satisfies $(2.6)$, completing the proof.

The set $\mathbf{A}(\tau ; f)$ is an affine subset of $\mathscr{B}$. A convenient representation of $\mathbf{A}$ can be found in terms of its underlying linear subspace and some particular element of $\mathscr{B}$. We consider the homogeneous problem in the tail associated with (2.1):

$$
\begin{aligned}
& \text { a) } \quad \frac{d v}{d x}=A(x) v, \quad x \in[\tau, \infty) \text {; } \\
& \text { b) } \quad \lim _{x \rightarrow \infty} B_{\infty} v(x)=0,
\end{aligned}
$$

and, if (2.1d) is imposed,

c) $\|v(x)\|$ bounded as $x \rightarrow \infty$.

We define $\mathscr{A}(\tau)$, the admissible space at $x=\tau$, as the set of all Cauchy data leading to solutions of (2.7). That is:

Definition 2.8. The set $\mathscr{A}(\tau) \subset \mathscr{B}$, the admissible space at $x=\infty$, is the set of all $v_{0} \in \mathscr{B}$ such that (2.7) has a solution satisfying:

$$
v(\tau)=v_{0}
$$

We note that $\mathscr{A}(\tau)$ is independent of the inhomogeneous term in (2.1). We further require a particular solution, $u_{p}(x)$, which satisfies:

$$
\begin{aligned}
& \text { a) } \frac{d u_{p}}{d x}=A(x) u_{p}+f(x), \quad x \in[\tau, \infty) \\
& \text { b) } \quad \lim _{x \rightarrow \infty} B_{\infty} u_{p}(x)=0
\end{aligned}
$$

and, if (2.1d) is imposed

$$
\text { c) } \quad\left\|u_{p}(x)\right\| \text { bounded as } x \rightarrow \infty \text {. }
$$

We note that if $\mathbf{A}(\tau ; f)$ is nonempty, at least one such $u_{p}(x)$ must exist. It is now possible to prove:

THEOREM 2.10. Let $u_{0} \in \mathscr{B}$. Then $u_{0} \in \mathbf{A}(\tau ; f)$ if and only if, for any particular solution $u_{p}(x)$

$$
u_{0}-u_{p}(\tau) \in \mathscr{A}(\tau)
$$

Proof. The proof is an immediate consequence of the definitions of $\mathbf{A}(\tau ; f), \mathscr{A}(\tau)$ and $u_{p}(x)$ combined with the linearity of $(2.1)$. 
If we assume that there exists a projection operator, $Q(\tau)$, into $\mathscr{A}(\tau)$, we can rewrite (2.10):

$$
(I-Q(\tau))\left(u_{0}-u_{p}(\tau)\right)=0
$$

In particular, the boundary condition, $(2.6 \mathrm{c})$, can be replaced by:

$$
(I-Q(\tau)) \omega(\tau)=(I-Q(\tau)) u_{p}(\tau)
$$

We emphasize that $u_{p}(x)$ can be any particular solution.

Finally, we write down a corollary of Theorem 2.6 which concerns the uniqueness of solutions.

Corollary. Suppose that for all $v_{0} \in \mathscr{A}(\tau)$ solutions to the Cauchy problem defined by (2.7a) and (2.8) are unique. Then (2.6) has a unique solution if and only if (2.1) does.

Proof. Assuming uniqueness of solutions to (2.1) immediately yields uniqueness for (2.6). In the other direction, note that the assumption above guarantees the uniqueness of $u_{+}(x)$ which, combined with the uniqueness of the finite interval solution, implies the uniqueness of $u$.

3. Solvability of the finite problem. In this section we assume that solutions to the homogeneous Cauchy problems:

$$
\begin{array}{ll}
\text { a) } & \frac{d v}{d x}=A(x) v, \quad x_{0} \leqq x \leqq x_{1} \quad \text { if } x_{1}>x_{0} ; \quad x_{1} \leqq x \leqq x_{0} \quad \text { if } x_{0}>x_{1} \\
\text { b) } & v\left(x_{0}\right)=v_{0}
\end{array}
$$

are unique for all $x_{0}, x_{1} \in[0, \infty)$. We define a solution operator $S\left(x_{1}, x_{0} ; A\right)$ in the following way:

Definition 3.2. Let $v_{0} \in \mathscr{B}$. If there exists a solution, $v(x)$ to problem (3.1) then

$$
S\left(x_{1}, x_{0} ; A\right) v_{0}=v\left(x_{1}\right)
$$

Otherwise, $v_{0}$ is said to be outside the domain of $S\left(x_{1}, x_{0} ; A\right)$.

The linearity of the differential equation implies the linearity of $s$. The stated uniqueness of solutions implies the consistency of the definition. Note that it is certainly necessary to restrict the domain of $S$ for ill-posed Cauchy problems such as those which arise in the study of elliptic equations. Whenever $S$ exists, however, it does have the familiar semi-group properties:

$$
\begin{array}{ll}
\text { a) } & S\left(x_{1}, x^{*} ; A\right) S\left(x^{*}, x_{0} ; A\right)=S\left(x_{1}, x_{0} ; A\right) \\
\text { b) } \quad & S\left(x_{0}, x_{0} ; A\right)=I .
\end{array}
$$

The notion of dichotomies is very useful in what follows. First we present definitions of exponential and ordinary dichotomies. These are adapted from Daletskiy and Krein [4], with some modifications required by the poosible nonexistence of solutions.

DEFINITION 3.4. We say that the problem

$$
\frac{d v}{d x}=A(x) v, \quad x \in[0, \infty)
$$


has an exponential dichotomy if, for any $x^{*} \in[0, \infty)$, the space $\mathscr{B}$ can be decomposed into a direct sum of subspaces $\mathscr{B}_{-}\left(x^{*}\right)$ and $\mathscr{B}_{+}\left(x^{*}\right)$ such that:

a) If $v \in \mathscr{B}_{-}\left(x^{*}\right)$ then, for some $N_{-}$and $\alpha_{-}>0$

i) $S\left(x, x^{*} ; A\right) v$ exists for any $x \geqq x^{*}$;

ii) $\left\|S\left(x, x^{*} ; A\right) v\right\| \leqq N_{-} e^{-\alpha_{-}\left(x-x^{*}\right)}\|v\|$.

b) If $v \in \mathscr{B}_{+}\left(x^{*}\right)$ then, for some $N_{+}$and $\alpha_{+}>0$

i) $S\left(x, x^{*} ; A\right) v$ exists for any $x \leqq x^{*}$;

ii) $\left\|S\left(x, x^{*} ; A\right) v\right\| \leqq N_{+} e^{-\alpha_{+}\left(x^{*}-x\right)}\|v\|$.

c) There exists $\gamma>0$, independent of $x^{*}$, such that

$$
\inf _{\substack{u_{ \pm} \in \mathscr{B}_{ \pm}\left(x^{*}\right) \\\left\|u_{ \pm}\right\|=1}}\left\|u_{+}+u_{-}\right\| \geqq \gamma .
$$

(This infimum is typically called the angular distance between $\mathscr{B}_{+}\left(x^{*}\right)$ and $\mathscr{B}_{-}\left(x^{*}\right)$.)

An ordinary dichotomy is defined as above except that $\alpha_{ \pm}=0$ is allowed. No "continuity" of the spaces as functions of $x^{*}$ has so far been required. In general, we impose a sort of continuity in the form of the following "no-mixing" condition.

DEFINITION 3.8. The dichotomy (3.5-3.7) satisfies the no-mixing condition if whenever

a) $\quad Q(x)$ is the projection operator into $\mathscr{B}_{-}(x)$,

b) $\quad S\left(x_{1}, x_{0} ; A\right) v$ exists

then

c) $\quad Q\left(x_{1}\right) S\left(x_{1}, x_{0} ; A\right) v=S\left(x_{1}, x_{0} ; A\right) Q\left(x_{0}\right) v$.

Assuming that the homogeneous problem has a dichotomy in the tail and that $\mathscr{B}_{-}(x)$ coincides with the admissible space, $\mathscr{A}(x)$, it is possible to write down an integral expression for a particular solution, $u_{p}(x)$ which is valid whenever $\|f(x)\|$ is integrable;

$$
u_{p}(x)=\int_{\tau}^{x} S(x, p ; A) Q(p) f(p) d p-\int_{x}^{\infty} S(x, p ; A)(I-Q(p)) f(p) d p .
$$

(The validity of (2.9a) follows from the direct differentiation of (3.9) while (2.9b) is insured by the identity of $\mathscr{B}_{-}(x)$ and $\mathscr{A}(x)$ combined with the absolute convergence of the integrals.) Note that it is always the case that $\mathscr{B}_{-}(x)=\mathscr{A}(x)$ if there is an exponential dichotomy. Then, only boundedness of $\|f\|$ need be assumed.

Formula (3.9) is extremely useful in the development of a perturbation theory. For now, we simply use it to write down a new expression for the boundary condition, (2.6c):

$$
(I-Q(\tau)) \omega(\tau)=-\int_{\tau}^{\infty} S(\tau, p)(I-Q(p)) f(p) d p
$$

Extending the dichotomy to the entire interval, we now can prove an existence theorem for the finite boundary value problem (2.6). 
THEOREM 3.11. Suppose that solutions to all Cauchy problems (3.1) are unique for $x_{0}, x_{1} \in[0, \tau]$ and that (3.1a) has a nonmixing ordinary dichotomy on $[0, \tau]$ with projector $Q(x)$ into $\mathscr{B}_{-}(x)$. Also assume that $\mathscr{B}_{-}(\tau)=\mathscr{A}(\tau)$. Then $(2.6)$ has a solution for arbitrary $f(x), u_{p}(\tau)$ and $\gamma_{0}$ in the range of $B_{0}$ if and only if the operator

$$
\Phi \omega=\left\{\begin{array}{c}
(I-Q(0)) \omega \\
B_{0} \omega
\end{array}\right\}
$$

has an inverse with domain containing all pairs of the form:

$$
\left(\begin{array}{l}
0 \\
\gamma
\end{array}\right), \quad \gamma \in \operatorname{Range}\left(B_{0}\right) \text {. }
$$

The solution is unique and bounded in terms of the inhomogeneous data if and only if this (restricted) inverse is.

Proof. We use the ordinary dichotomy defined by $Q(x)$ to solve certain initial value problems. Let

$$
\omega_{+}(x)=S(x, \tau ; A)(I-Q(\tau)) u_{p}(\tau)+\int_{\tau}^{x} S(x, p ; A)(I-Q(p)) f(p) d p .
$$

This exists for all $x$ on $[0, \tau]$ by the definition of $Q$. If we seek solutions to (2.6) in the form

$$
\omega(x)=\omega_{+}(x)+\omega_{-}(x)
$$

then $\omega$ is a solution if and only if $\omega_{-}$solves

$$
\begin{array}{ll}
\text { a) } & \frac{d \omega_{-}}{d x}=A(x) \omega_{-}+Q(x) f(x) \\
\text { b) } & B_{0} \omega_{-}(0)=\gamma_{0}-B_{0} \omega_{+}(0) \\
\text { c) } & (I-Q(\tau)) \omega_{-}(\tau)=0 .
\end{array}
$$

We write $\omega_{-}(x)$ in the form:

$$
\omega_{-}(x)=S(x, 0 ; A) \omega_{-}(0)+\int_{0}^{x} S(x, p ; A) Q(p) f(p) d p .
$$

The integral term again exists by the definition of $Q$ so that this representation is valid for any solution of (3.15a). By (3.15c) and (3.8c) we have:

$$
0=(I-Q(\tau)) \omega_{-}(\tau)=S(\tau, 0 ; A)(I-Q(0)) \omega_{-}(0) ;
$$

which, by the uniqueness of solutions to the Cauchy problem, implies

$$
(I-Q(0)) \omega_{-}(0)=0 .
$$

Hence, we can find a solution to (3.15) if and only if we can simultaneously solve:

$$
\begin{aligned}
& (I-Q(0)) \omega_{-}(0)=0 \\
& B_{0} \omega_{-}(0)=\gamma_{0}-B_{0} \omega_{+}(0)
\end{aligned}
$$

which in component form yields (3.11), completing the proof. 
Estimates of the solution in terms of the inhomogeneous data are now obtained from the explicit representation in terms of $\omega_{+}$and $\omega_{-}$. Assume that
a) $\quad\|S(x, p ; A) Q(p)\| \leqq K_{-}(x, p), \quad 0 \leqq p \leqq x \leqq \tau$;
b) $\|S(x, p ; A)(I-Q(p))\| \leqq K_{+}(x, p), \quad 0 \leqq x \leqq p \leqq \tau$;
c) $\left\|\Phi^{-1}\left(\begin{array}{l}0 \\ \gamma\end{array}\right)\right\| \leqq K_{\phi}\|\gamma\|, \quad \gamma \in \operatorname{Range}\left(B_{0}\right)$;
d) $\left\|B_{0}\right\| \leqq K_{0}$.

Then we have, directly estimating (3.13) and (3.16) and using the fact that $Q(0) \omega_{-}(0)=$ $\omega_{-}(0)$,

$$
\begin{aligned}
\|\omega(x)\| \leqq & K_{-}(x, 0)\left\|\gamma_{0}\right\|+\max _{x \in[0, \tau]}\|f(x)\| \int_{0}^{x} K_{-}(x, p) d p \\
& +\max _{x \in[0, \tau]}\|f(x)\|\left(\int_{x}^{\tau} K_{+}(x, p) d p+K_{-}(x, 0) K_{\phi} K_{0} \int_{0}^{\tau} K_{+}(0, p) d p\right) \\
& +\left\|u_{p}(\tau)\right\|\left(K_{+}(x, \tau)+K_{-}(x, 0) K_{\phi} K_{0} K_{+}(0, \tau)\right) .
\end{aligned}
$$

Equation (3.18) allows us to estimate the errors caused by approximations to $Q(\tau)$ and $u_{p}(\tau)$. Suppose we solve the following finite problem instead of (2.6):

$$
\begin{array}{ll}
\text { a) } & \frac{d \omega_{a}}{d x}=A(x) \omega_{a}+f(x), \quad 0 \leqq x \leqq \tau, \\
\text { b) } & B_{0} \omega_{a}(0)=\gamma_{0} ; \\
\text { c) } & \left(I-Q^{*}(\tau)\right) \omega_{a}(\tau)=\left(I-Q^{*}(\tau)\right) u_{p}^{*}(\tau)
\end{array}
$$

where $Q^{*}(\tau)$ and $u_{p}^{*}(\tau)$ differ from $Q(\tau)$ and $u_{p}(\tau)$. We define the error, $e(x)$, by

$$
e(x) \equiv \omega(x)-\omega_{a}(x)
$$

and find that it satisfies:

$$
\begin{array}{ll}
\text { a) } \quad \frac{d e}{d x}=A(x) e, & 0 \leqq x \leqq \tau, \\
\text { b) } \quad B_{0} e(0)=0, & \\
\text { c) } \quad(I-Q(\tau)) e(\tau)= & (I-Q(\tau))\left(u_{p}(\tau)-u_{p}^{*}(\tau)\right) \\
& +\left(Q(\tau)-Q^{*}(\tau)\right)\left(u_{p}^{*}(\tau)-\omega_{a}(\tau)\right) \\
\equiv & \Delta(t) .
\end{array}
$$

Note that $\Delta(\tau)$, by construction, is in the range of $I-Q(\tau)$. (We assume, of course, that $\omega_{a}(x)$ exists.) Therefore we have:

$$
(I-Q(\tau)) \Delta(\tau)=\Delta(\tau)
$$

We now plug into (3.18) to obtain:

$$
\|e(x)\| \leqq\left(K_{+}(x, \tau)+K_{-}(x, 0) K_{\phi} K_{0} K_{+}(0, \tau)\right)\|\Delta(\tau)\|
$$


Further specializing to the case of an exponential dichotomy this becomes:

$$
\|e(x)\| \leqq\left(N_{+} e^{\alpha_{+}(x-\tau)}+N_{-} e^{-\alpha_{-} x} K_{\phi} K_{0} N_{+} e^{-\alpha_{+} \tau}\right)\|\Delta(\tau)\| .
$$

That is, the large part of the error decays exponentially off the artificial boundary.

4. Problems with constant tails. In this section we restrict ourselves to problems which are autonomous in $x$ for $x$ sufficiently large. That is, we assume there exists $\tau$ such that:

$$
A(x) \equiv A_{\infty}, \quad x \geqq \tau .
$$

We also require that the constant coefficient problem in the tail be separable. That is, we require that a complete spectral representation be associated with $A_{\infty}$ :

Assumption 4.2. There exists a countable set of pairs, $\left(\lambda_{n}, u_{n}\right)$, with $\lambda_{n}$ a complex number, $u_{n} \in \mathscr{B}$ and 0 not an accumulation point of $\left\{\lambda_{n}\right\}$ and there exist adjoint pairs, $\left(\lambda_{n}^{*}, v_{n}\right)$, with $v_{n} \in \operatorname{Dual}(\mathscr{B})$, satisfying
i) $\quad A_{\infty} u_{n}=\lambda_{n} u_{n}$;
ii) $A_{\infty}^{*} v_{n}=\lambda_{n}^{*} v_{n}$;
iii) $\quad\left(v_{m}, u_{n}\right)=\delta_{m n}$.

Furthermore, any function $u \in \mathscr{B}$ can be uniquely written in the form:

$$
u=\sum_{n=1}^{\infty} c_{n} u_{n}, \quad c_{n}=\left(v_{n}, u\right) .
$$

Using the eigenfunction expansions defined above, it is easy to write down conditions for the existence of dichotomies for the constant problem as well as representations of the various operators discussed in the preceding sections. In particular we have the following theorem, whose proof follows immediately from the (formal) solution of the Cauchy problem in terms of the eigenfunction expansions. (For the details of these see Hagstrom [7].)

THEOREM 4.4. a) If all eigenvalues, $\lambda_{n}$, of $A_{\infty}$ are bounded away from the imaginary axis, then the homogeneous problem associated with $A_{\infty}$ has an exponential dichotomy with spaces

$$
\begin{aligned}
& \mathscr{B}_{+} \equiv \operatorname{span}\left\{u_{i}: \operatorname{Re} \lambda_{i}>0\right\} \\
& \mathscr{B}_{-} \equiv \operatorname{span}\left\{u_{i}: \operatorname{Re} \lambda_{i}<0\right\} .
\end{aligned}
$$

The exponents, $\alpha_{ \pm}$, are given by:

$$
\begin{aligned}
& \alpha_{+}=\underset{\operatorname{Re} \lambda_{i}>0}{\text { g.1.b. }}\left|\operatorname{Re} \lambda_{i}\right| ; \\
& \alpha_{-}=\underset{\operatorname{Re} \lambda_{i}<0}{\text { g.1.b. }}\left|\operatorname{Re} \lambda_{i}\right| .
\end{aligned}
$$

b) Let $\mathscr{B}_{ \pm}$be defined as above and let $\mathscr{B}_{0}$ be given by:

$$
\mathscr{B}_{0} \equiv \operatorname{span}\left\{u_{i}: \operatorname{Re} \lambda_{i}=0\right\} \text {. }
$$

Let $\mathscr{B}_{0}^{+} \oplus \mathscr{B}_{0}^{-}$be any direct sum decomposition of $\mathscr{B}_{0}$. Then an ordinary dichotomy is induced by the spaces $\mathscr{B}_{+} \oplus \mathscr{B}_{0}^{+}$and $\mathscr{B}_{-} \oplus \mathscr{B}_{0}^{-}$. 
We note that by the conclusions of part (b), there can be many ordinary dichotomies associated with a problem whose operator has eigenvalues with zero real part. Which of these is the right one to use for the boundary condition depends on the boundary operator at infinity, $B_{\infty}$. Representations of the solution operator, $S$, are also easy to obtain.

The theorem above can be applied to the example of $\S 1$, problem (1.1). Rewriting the problem in first order form according to transformation (2.4), the operator $A_{\infty}$ is given by:

$$
A_{\infty}=\left(\begin{array}{cc}
0 & -\nabla_{\mathbf{y}}^{2}-a_{\infty} \\
1 & 0
\end{array}\right) .
$$

Its eigenvalues are given by $\pm \lambda_{n}$ and $\pm i \alpha_{n}$, defined by the reduced eigenvalue problem (1.3) through equation (1.4). If (1.3) had no positive eigenvalues, the problem in the tail would have an exponential dichotomy. In the case of an ordinary dichotomy, the boundary condition (1.7a) corresponds to the choice:

$$
\mathscr{B}_{0}^{+} \equiv \mathscr{B}_{0}, \quad \mathscr{B}_{0}^{-} \equiv \varnothing .
$$

If, instead of (1.1d), some other condition was imposed (for example a radiation condition) this choice would change. We note that using the integral representation of the boundary condition, (3.10), the condition that the inhomogeneous term vanish in the tail can be replaced by an integrability assumption. The boundary condition, (1.7), is then replaced by:

$$
\frac{1}{2}\left(\begin{array}{cc}
1 & \lambda_{n} \\
\frac{1}{\lambda_{n}} & 1
\end{array}\right)\left(\begin{array}{c}
c_{n}^{\prime}(\tau) \\
c_{n}(\tau)
\end{array}\right)=-\frac{1}{2} \int_{\tau}^{\infty} d s e^{-\lambda_{n}(s-\tau)}\left(\begin{array}{cc}
1 & \lambda_{n} \\
\frac{1}{\lambda_{n}} & 1
\end{array}\right)\left(\begin{array}{c}
f_{n}(s) \\
0
\end{array}\right)
$$

which implies

$$
c_{n}^{\prime}(\tau)=-\lambda_{n} c_{n}(\tau)-\int_{\tau}^{\infty} d s e^{-\lambda_{n}(s-\tau)} f_{n}(s), \quad n=m+1, m+2, \cdots
$$

For the imaginary eigenvalues we have:

$$
\left(\begin{array}{c}
c_{n}^{\prime}(\tau) \\
c_{n}(\tau)
\end{array}\right)=-\frac{1}{2} \int_{\tau}^{\infty} d s\left\{e^{-i \alpha_{n}(s-\tau)}\left(\begin{array}{cc}
1 & i \alpha_{n} \\
\frac{1}{i \alpha_{n}} & 1
\end{array}\right)+e^{i \alpha_{n}(s-\tau)}\left(\begin{array}{cc}
1 & -i \alpha_{n} \\
\frac{1}{i \alpha_{n}} & 1
\end{array}\right)\right\}\left(\begin{array}{c}
f_{n}(s) \\
0
\end{array}\right)
$$

which implies

$$
\begin{aligned}
& c_{n}^{\prime}(\tau)=-\int_{\tau}^{\infty} \cos \left[\alpha_{n}(s-\tau)\right] f_{n}(s) d s \\
& c_{n}(\tau)=\frac{1}{\alpha_{n}} \int_{\tau}^{\infty} \sin \left[\alpha_{n}(s-\tau)\right] f_{n}(s) d s
\end{aligned}
$$

For a general partial differential equation with a constant tail, the eigenvalue problem of its operator, $A_{\infty}$, can be reduced to an eigenvalue problem for a partial differential operator. In particular, it's eigenvalues, $\lambda$, correspond to solutions of:

$$
\left[\sum_{j=0}^{n} P_{j}\left(\mathbf{y}, \frac{\partial}{\partial \mathbf{y}}\right) \lambda^{j}\right] Y(\mathbf{y})=0
$$


coupled with the appropriate boundary conditions. This is the eigenvalue problem associated with the Laplace transform in $x$ of the equation in the tail. We note that in practice it is the reduced eigenvalue problem, (4.9), which we suggest be solved to obtain the boundary conditions. The reduction to first order form is made in an effort to simplify the theory. The use of (4.9) to derive boundary conditions was first suggested by Gustafsson and Kreiss [6].

The completeness of the eigenfunctions of $A_{\infty}$ depends on the completeness of the eigenfunctions of (4.9). This property does not hold in general and is difficult to check. For a class of elliptic and parabolic problems, Agmon and Nirenberg [1, Thm. 5.8] establish the completeness of the eigenfunctions and generalized eigenfunctions of (4.9) whose eigenvalues have negative real part in the class of solutions which are absolutely integrable along with their first $n-1 x$ derivatives. In this case, the solution of (4.9) is guaranteed to yield a representation of the admissible space.

5. Perturbation theory and asymptotic boundary conditions. In the preceding section we found useful representations of the projection operator, $Q(\tau)$, of the admissible space and of the particular solution, $u_{p}(x)$ for equations of the form (2.1) with constant tails. In the present section we relax this assumption and replace it with:

$$
\lim _{x \rightarrow \infty} A(x)=A_{\infty}
$$

Equivalently we write:

$$
A(x)=A_{\infty}+B(x), \quad \lim _{x \rightarrow \infty}\|B(x)\|=0 .
$$

Assuming $A_{\infty}$ has a dichotomy, it is possible to make an asymptotic analysis of the perturbed problem defined by $A(x)$. In particular, we obtain representations of the projector, $Q(\tau)$, into the admissible space. Consider the homogeneous problem in the tail:

$$
\begin{aligned}
& \text { a) } \quad \frac{d v}{d x}=A_{\infty} v+B(x) v, x \geqq \tau ; \\
& \text { b) } \quad \lim _{x \rightarrow \infty} B_{\infty} v(x)=0 ; \\
& \text { c) } \quad\|u(x)\| \text { bounded as } x \rightarrow \infty .
\end{aligned}
$$

Treating $B(x) v$ as an inhomogeneous term, we have, by (3.10), that $v(x)$ must satisfy:

$$
\left(I-Q_{\infty}(\tau)\right) v(\tau)=-\int_{\tau}^{\infty} S\left(\tau, p ; A_{\infty}\right)\left(I-Q_{\infty}(p)\right) B(p) v(p) d p
$$

Also, from (3.10), we have a representation of $v$ which must be valid if $v$ exists;

$$
\begin{aligned}
v(x)= & S\left(x, \tau ; A_{\infty}\right) Q_{\infty}(\tau) v(\tau)+\int_{\tau}^{x} S\left(x, p ; A_{\infty}\right) Q_{\infty}(p) B(p) v(p) d p \\
& -\int_{x}^{\infty} S\left(x, p ; A_{\infty}\right)\left(I-Q_{\infty}(p)\right) B(p) v(p) d p .
\end{aligned}
$$

Let any $\xi_{0} \in \mathscr{A}_{\infty}(\tau)$ be given and replace $Q_{\infty}(\tau) v(\tau)$ in (5.5) by $\xi_{0}$. If the following condition holds:

$$
\begin{aligned}
\sup _{x \geqq \tau} \|\left[\int_{\tau}^{x} S\left(x, p ; A_{\infty}\right) Q_{\infty}(p) B(p) d p\right. \\
\left.-\int_{x}^{\infty} S\left(x, p ; A_{\infty}\right)\left(I-Q_{\infty}(p)\right) B(p) d p\right] \|=K<1
\end{aligned}
$$


then the contraction mapping theorem can be used to establish the existence of a unique bounded solution to equation (5.5), $v\left(x ; \xi_{0}\right)$. Furthermore, we clearly have that:

$$
\begin{aligned}
& Q_{\infty} v\left(\tau ; \xi_{0}\right)=\xi_{0} \\
& \left(I-Q_{\infty}(\tau)\right) v\left(\tau ; \xi_{0}\right)=-\int_{\tau}^{\infty} S\left(\tau, p ; A_{\infty}\right)\left(I-Q_{\infty}(p)\right) B(p) v\left(p ; \xi_{0}\right) d p .
\end{aligned}
$$

Hence, whenever (5.6) is valid, we can find, for any $\xi_{0} \in \mathscr{A}_{\infty}(\tau)$, a unique element, $v\left(\tau ; \xi_{0}\right)$, of $\mathscr{A}(\tau)$. A projector into $\mathscr{A}(\tau)$ is given implicitly by $(5.7)$ :

$$
Q(\tau) \xi=Q_{\infty}(\tau) \xi-\int_{\tau}^{\infty} d p S\left(\tau, p ; A_{\infty}\right)\left(I-Q_{\infty}(p)\right) B(p) v\left(p ; Q_{\infty}(\tau) \xi\right)
$$

These conditions lead us to the following theorem:

THEOREM 5.9. We suppose that either the unperturbed problem has an ordinary dichotomy and $\|B(x)\|$ is integrable or that the unperturbed problem has an exponential dichotomy. Then, for $\tau$ sufficiently large, a unique solution, $v\left(x ; \xi_{0}\right)$, exists for any $\xi_{0} \in \mathscr{A}_{\infty}(\tau)$ and (5.8) is valid.

Proof. It is only necessary to satisfy (5.6). In the first case we have:

$$
K \leqq\left(N_{+}+N_{-}\right) \int_{\tau}^{\infty}\|B(x)\| d x
$$

while in the second we have:

$$
K \leqq\left(\frac{N_{+}}{\alpha_{+}}+\frac{N_{-}}{\alpha_{-}}\right) \max _{x \geqq \tau}\|B(x)\| .
$$

For both cases, the assumptions on $B$ allow us to make the right-hand sides arbitrarily small by choosing $\tau$ sufficiently large, completing the proof.

The contraction mapping solution of (5.5) leads to a natural iterative procedure for the approximation of $v\left(x ; \xi_{0}\right)$ and, ultimately, of the operator $Q$. We let:

$$
\begin{aligned}
v^{(0)}\left(x ; \xi_{0}\right)=S\left(x, \tau ; A_{\infty}\right) \xi_{0} & \\
v^{(n+1)}\left(x ; \xi_{0}\right)= & v^{(0)}\left(x ; \xi_{0}\right)+\int_{\tau}^{x} d p S\left(x, p ; A_{\infty}\right) Q_{\infty}(p) B(p) v^{(n)}\left(p ; \xi_{0}\right) \\
& -\int_{x}^{\infty} d p S\left(x, p ; A_{\infty}\right)\left(I-Q_{\infty}(p)\right) B(p) v^{(n)}\left(p ; \xi_{0}\right)
\end{aligned}
$$

Then, by the contraction estimates:

$$
\left\|v^{(n)}\left(x ; \xi_{0}\right)-v\left(x ; \xi_{0}\right)\right\| \leqq \frac{K^{n+1}}{1-K}\left\|v^{(0)}\left(x ; \xi_{0}\right)\right\|
$$

We define our $n$th approximation to $A(\tau), Q^{(n)}(\tau)$, by:

$$
Q^{(n)}(\tau) \xi=Q_{\infty}(\tau) \xi-\int_{\tau}^{\infty} d p S\left(\tau, p ; A_{\infty}\right)\left(I-Q_{\infty}(p)\right) B(p) v^{(n-1)}\left(p ; Q_{\infty}(\tau) \xi\right)
$$

The error due to this approximation is estimated by:

$$
\left\|Q(\tau) \xi-Q^{(n)}(\tau) \xi\right\| \leqq \frac{K^{n+1}}{1-K}\left\|v^{(0)}\left(x ; Q_{\infty}(\tau) \xi\right)\right\| .
$$

(Note: in all cases the norm of a $\mathscr{B}$-valued function of $x$ is taken to be the maximum in $x$ of its $\mathscr{B}$ norms.) 
We now apply these results to the case when the constant tail problem has an exponential dichotomy and $A_{\infty}$ has a complete spectrum. We assume that $B(x)$ has an expansion of the form:

$$
B(x)=\frac{1}{x} B^{(1)}+\frac{1}{x^{2}} B^{(2)}+\cdots .
$$

(The expansions could easily be carried out for more general forms.) Plugging into the formulas above we have:

$$
\begin{aligned}
V^{(1)}(x ; \xi)= & \sum_{\substack{n \\
\operatorname{Re} \lambda_{n}<0}} c_{n} e^{\lambda_{n}(x-\tau)}+\sum_{\substack{n \\
\operatorname{Re} \lambda_{n}^{<}<0}} \sum_{\substack{m \\
\operatorname{Re} \lambda_{m}<0}} \int_{\tau}^{x} d p e^{\lambda_{n}(x-p)} B_{n m}(p) c_{m} e^{\lambda_{m}(p-\tau)} \\
& -\sum_{\substack{n \\
\operatorname{Re} \lambda_{n}^{n}>0}} \sum_{\substack{m \\
\operatorname{Re} \lambda_{m}<0}} \int_{x}^{\infty} d p e^{\lambda_{n}(x-p)} B_{n m} l(p) c_{m} e^{\lambda_{m}(p-\tau)}
\end{aligned}
$$

where

$$
c_{n}=\left(v_{n}, \xi\right), \quad B_{n m}(x)=\left(v_{n}, B(x) u_{m}\right)
$$

Using (5.13) and approximating the integrals using integration by parts yields to within an $O\left(1 / \tau^{2}\right)$ error:

$$
\begin{aligned}
v^{(1)}(x ; \xi)= & \sum_{\substack{n \\
\operatorname{Re} \lambda_{n}<0}} u_{n} c_{n} e^{\lambda_{n}(x-\tau)}+\sum_{\substack{n \\
\operatorname{Re} \lambda_{n}^{<}<n}} \sum_{\substack{m \\
\operatorname{Re} \lambda_{m}^{<}<0 \\
\lambda_{m} \neq \lambda_{n}}} u_{n} \frac{B_{n m}^{(1)}}{\lambda_{m}-\lambda_{n}} c_{m}\left(\frac{e^{\lambda_{m}(x-\tau)}}{x}-\frac{e^{\lambda_{n}(x-\tau)}}{\tau}\right) \\
& +\sum_{\substack{n \\
\operatorname{Re} \lambda_{n}<0}} u_{n} B_{n m}^{(1)} c_{n} e^{\lambda_{n}(x-\tau)} \log \left(\frac{x}{\tau}\right)+\sum_{\substack{n \\
\operatorname{Re} \lambda_{n}>0 \operatorname{Re} \lambda_{m}<0}} \sum_{n} \frac{B_{n m}^{(1)}}{\lambda_{m}-\lambda_{n}} c_{m} \frac{e^{\lambda_{m}(x-\tau)}}{x}
\end{aligned}
$$

Putting this expression into (5.11) and approximating the integrals in a similar fashion yields:

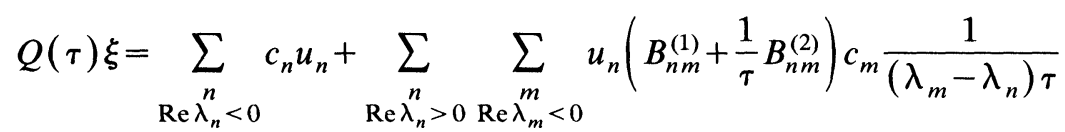

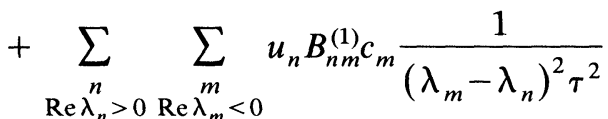

$$
\begin{aligned}
& -\sum_{\substack{n \\
\operatorname{Re} \lambda_{n}>0}} \sum_{\substack{j \\
\operatorname{Re} \lambda_{j}<0}} \sum_{\substack{m \\
\operatorname{Re} \lambda_{m}<0}} u_{n} B_{n j}^{(1)} B_{j m}^{(1)} c_{m} \frac{1}{\tau^{2}\left(\lambda_{m}-\lambda_{n}\right)\left(\lambda_{j}-\lambda_{n}\right)} \\
& +\sum_{\operatorname{Re} \lambda_{n}^{n}>0} \sum_{\substack{j \\
\operatorname{Re} \lambda_{j}>0}} \sum_{\substack{m \\
\operatorname{Re} \lambda_{m}^{<}<0}} u_{n} B_{n j}^{(1)} B_{j m}^{(1)} c_{m} \frac{1}{\tau^{2}\left(\lambda_{m}-\lambda_{j}\right)\left(\lambda_{m}-\lambda_{n}\right)}+O\left(\frac{1}{\tau^{3}}\right) \\
& c_{n}=\left(v_{n}, \xi\right) \text {. }
\end{aligned}
$$


The generality of the expansion given above makes its automatic computation a real possibility. Note that the expansion is equivalent to the one obtained by Jepson and Keller [10] for ordinary differential equations.

Formula (5.17) can be applied to the Laplacian example, (1.1), where the potential $a(\mathbf{y})$ is replaced by:

$$
a(x, \mathbf{y})=a_{0}(\mathbf{y})+\frac{1}{x} a_{1}(\mathbf{y})+\frac{1}{x^{2}} a_{2}(\mathbf{y})+\cdots .
$$

Then, the matrix elements $B_{n m}^{(i)}$ are given by:

$$
B_{n m}^{(i)}=\frac{1}{2 \lambda_{n}} \int_{\Omega} d \mathbf{y} Y_{n}(\mathbf{y}) Y_{m}(\mathbf{y}) a_{i}(\mathbf{y}) .
$$

Expansions of a particular solution can be derived in a similar manner. Let $u_{\infty}(x)$ be any particular solution of the unperturbed problem. Then, a solution of the integral equation:

$$
\begin{aligned}
u(x)= & u_{\infty}(x)+\int_{\tau}^{x} 4 k S(x, p) Q_{\infty}(p) B(p) u(p) d p \\
& -\int_{x}^{\infty} S(x, p)\left(I-Q_{\infty}(p)\right) B(p) u(p) d p
\end{aligned}
$$

is a particular solution of the perturbed problem. Given the inequality (5.6), a unique bounded solution of (5.20) exists by the contraction mapping theorem. It can be approximated by an iterative process analogous to the one described by (5.9). Perturbations of the inhomogeneous term could also be included.

Finally, we note that (5.17) is valid for some problems which do not satisfy (5.6). An important example is afforded by the exterior Helmholtz problem in two dimensions. The equation in the tail is:

$$
\frac{\partial^{2} u}{\partial r^{2}}+\frac{1}{r} \frac{\partial u}{\partial r}+\frac{1}{r^{2}} \frac{\partial^{2} u}{\partial \theta^{2}}+k^{2} u=0, \quad r \in[\tau, \infty), \quad \theta \in[0,2 \pi)
$$

together with boundary conditions

a) $u$ periodic in $\theta$;

b) $\quad \lim _{r \rightarrow \infty} r^{1 / 2}\left(\frac{\partial u}{\partial r}-i k u\right)=0$.

Rewritten in first order form these become:

a) $\frac{\partial}{\partial r}\left(\begin{array}{l}\omega \\ u\end{array}\right)+\left(\begin{array}{cc}0 & k^{2} \\ -1 & 0\end{array}\right)\left(\begin{array}{l}\omega \\ u\end{array}\right)+\frac{1}{r}\left(\begin{array}{ll}1 & 0 \\ 0 & 0\end{array}\right)\left(\begin{array}{l}\omega \\ u\end{array}\right)$

$$
+\frac{1}{r^{2}}\left(\begin{array}{cc}
0 & \frac{\partial^{2}}{\partial \theta^{2}} \\
0 & 0
\end{array}\right)\left(\begin{array}{l}
\omega \\
u
\end{array}\right)=\left(\begin{array}{l}
0 \\
0
\end{array}\right) ;
$$

b) $\left(\begin{array}{l}\omega \\ u\end{array}\right)$ periodic in $\theta$;

c) $\quad \lim _{r \rightarrow \infty} r^{1 / 2}(\omega-i k u)=0$. 
There are two obstacles to the application of the preceding theory to problem (5.23). The first is that the perturbation

$$
\frac{1}{r^{2}}\left(\begin{array}{cc}
0 & \frac{\partial^{2}}{\partial \theta^{2}} \\
0 & 0
\end{array}\right)
$$

is apparently unbounded. The second is that the perturbation

$$
\frac{1}{r}\left(\begin{array}{ll}
1 & 0 \\
0 & 0
\end{array}\right)
$$

is nonintegrable while the limiting equation,

$$
\frac{\partial}{\partial r}\left(\begin{array}{l}
\omega \\
u
\end{array}\right)+\left(\begin{array}{cc}
0 & k^{2} \\
-1 & 0
\end{array}\right)\left(\begin{array}{l}
\omega \\
u
\end{array}\right)=\left(\begin{array}{l}
0 \\
0
\end{array}\right)
$$

has an ordinary rather than an exponential dicohotomy. Nonetheless, it is possible to apply formula (5.17), or any higher order approximation to the boundary condition, to this problem. (It is necessary, of course, to identify the imaginary eigenvalue $i k$ with eigenvalues with negative real part and $-i k$ with eigenvalues with positive real part when applying the formulas.) The resulting boundary condition is:

$$
\begin{aligned}
(I-Q(\tau))\left(\begin{array}{c}
\omega(\tau) \\
u(\tau)
\end{array}\right)= & \frac{1}{2}\left(\begin{array}{cc}
1 & -i k \\
-\frac{1}{i k} & 1
\end{array}\right)\left(\begin{array}{c}
\omega(\tau) \\
u(\tau)
\end{array}\right) \\
& -\frac{1}{2 i k \tau}\left(\frac{1}{2}+\frac{1}{2 i k \tau} \frac{\partial^{2}}{\partial \theta^{2}}\right)\left(\begin{array}{cc}
-\frac{1}{2} & \frac{-i k}{2} \\
\frac{1}{2 i k} & \frac{1}{2}
\end{array}\right)\left(\begin{array}{l}
\omega(\tau) \\
u(\tau)
\end{array}\right) \\
& +\frac{1}{4 k^{2} \tau^{2}} \cdot \frac{1}{2}\left(\begin{array}{cc}
-\frac{1}{2} & \frac{-i k}{2} \\
\frac{1}{2 i k} & \frac{1}{2}
\end{array}\right)\left(\begin{array}{l}
\omega(\tau) \\
u(\tau)
\end{array}\right) \\
& +\frac{1}{4 k^{2} \tau^{2}} \cdot \frac{1}{4}\left(\begin{array}{cc}
-\frac{1}{2} & \frac{-i k}{2} \\
\frac{1}{2 i k} & \frac{1}{2}
\end{array}\right)\left(\begin{array}{l}
\omega(\tau) \\
u(\tau)
\end{array}\right) \\
& -\frac{1}{4 k^{2} \tau^{2}} \cdot \frac{1}{4}\left(\begin{array}{cc}
-\frac{1}{2} & \frac{-i k}{2} \\
\frac{1}{2 i k} & \frac{1}{2}
\end{array}\right)\left(\begin{array}{l}
\omega(\tau) \\
u(\tau)
\end{array}\right)+O\left(\frac{1}{\tau^{3}}\right)=\left(\begin{array}{l}
0 \\
0
\end{array}\right)
\end{aligned}
$$

which can be written:

$$
\frac{\partial u}{\partial r}(\tau, \theta)=i k u(\tau, \theta)-\frac{1}{2 \tau} u(\tau, \theta)-\frac{1}{2 i k \tau^{2}} \frac{\partial^{2} u}{\partial \theta^{2}}(\tau, \theta)-\frac{1}{8 i k \tau^{2}} u(\tau, \theta) .
$$

The validity of (5.26) can be established by other means. See, for example, Bayliss, Gunzburger and Turkel [2]. We note that the error depends on higher $\theta$ derivatives of $u$. 
6. Nonlinear problems. In this final section we apply the perturbation theory to nonlinear problems. We restrict ourselves to abstract problems of the form:
a) $\quad \frac{d u}{d x}=F(u), \quad x \geqq \tau$;
b) $\quad \lim _{x \rightarrow \infty} u(x)=u_{\infty}$;
c) $\quad F\left(u_{\infty}\right)=0$

where $u(x)$ is an element of some Banach space, $\mathscr{B}$, and $F$ is a nonlinear operator with domain and range in $\mathscr{B}$. Letting $v=u-u_{\infty}$, we rewrite (6.1):
a) $\quad \frac{d v}{d x}=F_{u}\left(u_{\infty}\right) v+R(v), \quad x \geqq \tau ;$
b) $\quad \lim _{x \rightarrow \infty} v(x)=0$;
c) $\quad R(v) \equiv F\left(u_{\infty}+v\right)-F_{u}\left(u_{\infty}\right) v$.

One approach to the solution of (6.1) or (6.2) would be Newton's method. Then, the theory of the preceding sections could be applied at each stage of the iteration. We, however, choose to work directly with (6.2), deriving exact boundary conditions which can be approximated by the methods of $\S 5$.

We generalize the notion of an admissible set (Definition 2.5) to be applicable to (6.2). Note that it is no longer an affine subset of $\mathscr{B}$. Central to our analysis is the behavior of solutions to the linearized problem in the tail:

$$
\frac{d \omega}{d x}=F_{u}\left(u_{\infty}\right) \omega, \quad x \geqq \tau .
$$

Treating the nonlinearity, $R(v)$, as an inhomogeneous term leads to the following equations for $v$, which are analogous to (5.4) and (5.5);

$$
\begin{aligned}
\left(I-Q_{\infty}(\tau)\right) v(\tau)=-\int_{\tau}^{\infty} S\left(\tau, p ; F_{u}\left(u_{\infty}\right)\right)\left(I-Q_{\infty}(p)\right) R(v(p)) d p \\
v(x)=S\left(x, \tau ; F_{u}\left(u_{\infty}\right)\right) Q_{\infty}(\tau) v(\tau)+\int_{\tau}^{x} S\left(x, p ; F_{u}\left(u_{\infty}\right)\right) Q_{\infty}(p) R(v(p)) d p \\
\quad-\int_{x}^{\infty} S\left(x, p ; F_{u}\left(u_{\infty}\right)\right)\left(I-Q_{\infty}(p)\right) R(v(p)) d p
\end{aligned}
$$

Here, $Q_{\infty}$ projects into the admissible space of the linearized problem (6.3). As in the linear case, the condition that (6.4) and (6.5) be simultaneously solvable is viewed as a condition for the admissibility of $v(\tau)$.

Following the derivation for the linear problem, we let $\xi_{0} \in \mathscr{A}_{\infty}(\tau)$ be given and use a contraction argument to establish the existence of a solution to the integral equation, $(6.5)$, with $Q_{\infty}(\tau) v(\tau)$ replaced by $\xi_{0}$. Due to the nonlinearity, some additional assumptions are needed: then

Assumption 6.6. a) There exists $\delta>0$ such that if $u_{1}, u_{2} \in \mathscr{B}$ and $\left\|u_{i}\right\| \leqq \delta, i=1,2$,

$$
\begin{gathered}
\sup _{x \geqq \tau} \| \int_{\tau}^{x} S\left(x, p ; F_{u}\left(u_{\infty}\right)\right) Q_{\infty}(p)\left(R\left(u_{1}\right)-R\left(u_{2}\right)\right) d p \\
-\int_{x}^{\infty} S\left(x, p ; F_{u}\left(u_{\infty}\right)\right)\left(I-Q_{\infty}(p)\right)\left(R\left(u_{1}\right)-R\left(u_{2}\right)\right) d p \| \\
\leqq K\left\|u_{1}-u_{2}\right\|, \quad K<1 .
\end{gathered}
$$


b) There exists $\delta_{1}>0$ such that if $u \in \mathscr{B}$ and $\|u\|<\delta$, then

$$
\begin{aligned}
\sup _{x \geqq \tau} \| \int_{\tau}^{x} S\left(x, p ; F_{u}\left(u_{\infty}\right)\right) Q_{\infty}(p) R(u) d u \\
\quad-\int_{x}^{\infty} S\left(x, p ; F_{u}\left(u_{\infty}\right)\right)\left(I-Q_{\infty}(p)\right) R(u) d p \| \leqq \delta-\delta_{1} .
\end{aligned}
$$

c)

$$
\sup _{x \geq \tau}\left\|S\left(x, \tau ; F_{u}\left(u_{\infty}\right)\right) \xi_{0}\right\|<\delta_{1}
$$

Given these, a solution to (6.5) is guaranteed by the contraction mapping theorem. Denoting this solution by $v\left(x ; \xi_{0}\right)$, an exact boundary condition, valid for small boundary data, can be written down from (6.4):

$$
\left(I-Q_{\infty}(\tau)\right) v(\tau)=-\int_{\tau}^{\infty} S\left(\tau, p ; F_{u}\left(u_{\infty}\right)\right)\left(I-Q_{\infty}(p)\right) R\left(v\left(p ; Q_{\infty}(\tau) v(\tau)\right)\right) d p .
$$

An approximation to (6.6) can be obtained from an iterative approximation to the solution of (6.5):

$$
\begin{aligned}
& \text { a) } v^{(0)}\left(x ; \xi_{0}\right)=S\left(x, \tau ; F_{u}\left(u_{\infty}\right)\right) \xi_{0} \text {, } \\
& \text { b) } v^{(n+1)}\left(x ; \xi_{0}\right)=v^{(0)}\left(x ; \xi_{0}\right)+\int_{\tau}^{x} S\left(x, p ; F_{u}\left(u_{\infty}\right)\right) Q_{\infty}(p) R\left(v^{(n)}\left(p ; \xi_{0}\right)\right) d p \\
& -\int_{x}^{\infty} S\left(x, p ; F_{u}\left(u_{\infty}\right)\right)\left(I-Q_{\infty}(p)\right) R\left(v^{(n)}\left(p ; \xi_{0}\right)\right) d p .
\end{aligned}
$$

The $n$th approximation to the boundary condition is, then, given by:

$$
\left(I-Q_{\infty}(\tau)\right) v(\tau)=-\int_{\tau}^{\infty} d p S\left(\tau, p ; F_{u}\left(u_{\infty}\right)\right)\left(I-Q_{\infty}(p)\right) R\left(v^{(n)}\left(p ; Q_{\infty}(\tau) v(\tau)\right)\right) .
$$

Error estimates follow as in the linear case and will be proportional to $K^{n+1}\left\|v^{(0)}\right\|$ which, in turn, we expect to be proportional to $\|v(\tau)\|^{n+2}$. Note that $R$ will often be given as an expansion:

$$
R(v) \sim \frac{1}{2} F_{u u}\left(u_{\infty}\right) v v+\frac{1}{6} F_{u u u}\left(u_{\infty}\right) v v v+\cdots .
$$

We take as many terms in this expansion when evaluating the integrals as is consistent with the number of terms in (6.7) we intend to retain.

Assume now that the linearized operator, $F_{u}\left(u_{\infty}\right)$, has a complete spectrum. Then, in order to satisfy part (a) of assumption (6.6), it is necessary to assume that there is an exponential dichotomy. From (6.9) we derive the following representation of $R(v)$ in terms of the eigenfunctions of $F_{u}\left(u_{\infty}\right)$ :

$$
\begin{aligned}
& \text { a) } \quad v=\sum_{n=1}^{\infty} c_{n} u_{n}, c_{n}=\left(v_{n}, u\right) \\
& \text { b) } \\
& \quad R(v)=\sum_{n=1}^{\infty} \gamma_{n}(v) u_{n}, \gamma_{n}(v) \sim \sum_{i, j} \alpha_{i j}^{(n)} c_{i} c_{j}+\sum_{i, j, k} \beta_{i j k}^{(n)} c_{i} c_{j} c_{k}+\cdots \\
& \text { c) } \quad \alpha_{i j}^{(n)}=\left(v_{n}, \frac{1}{2} F_{u u} u_{i} u_{j}\right), \beta_{i j k}^{(n)}=\left(v_{n}, \frac{1}{6} F_{u u u} u_{i} u_{j} u_{k}\right), \cdots
\end{aligned}
$$


The function $v^{(1)}(x ; \xi)$ is given by:

$$
\begin{aligned}
& v^{(1)}(x ; \xi)=\sum_{\substack{n \\
\operatorname{Re} \lambda_{n}<0}} u_{n} c_{n} e^{\lambda_{n}(x-\tau)} \\
& +\sum_{\substack{n \\
\operatorname{Re} \lambda_{n}<0}} \sum_{\substack{i \\
\operatorname{Re} \lambda_{i}<0 \\
\lambda_{i}+\lambda_{j} \neq \lambda_{n}}} \sum_{\substack{j \\
\operatorname{Re} \lambda_{j}<0}} u_{n} \alpha_{i j}^{n} \frac{c_{i} c_{j}}{\left(\lambda_{i}+\lambda_{j}-\lambda_{n}\right)}\left(e^{\left(\lambda_{i}+\lambda_{j}\right)(x-\tau)}-e^{\lambda_{n}(x-\tau)}\right) \\
& +\sum_{\substack{n \\
\operatorname{Re} \lambda_{n}<0}} \sum_{\substack{i \\
\operatorname{Re} \lambda_{i}<0 \\
\lambda_{i}+\lambda_{j}=\lambda_{n}}} \sum_{\substack{j \\
\operatorname{Re} \lambda_{j}<0}} u_{n} \alpha_{i j}^{n} c_{i} c_{j} e^{\lambda_{n}(x-\tau)}(x-\tau) \\
& +\sum_{\substack{n \\
\operatorname{Re} \lambda_{n}>0}} \sum_{\substack{i \\
\operatorname{Re} \lambda_{i}<0}} \sum_{\substack{j \\
\operatorname{Re} \lambda_{j}<0}} u_{n} \alpha_{i j}^{n} c_{i} c_{j} \frac{1}{\lambda_{i}+\lambda_{j}-\lambda_{n}} e^{\left(\lambda_{i}+\lambda_{j}\right)(x-\tau)}+O\left(\|\xi\|^{3}\right) ; \\
& c_{n}=\left(v_{n}, \xi\right) \text {. }
\end{aligned}
$$

This yields the following approximation to the boundary condition, which we write in terms of the expansion coefficients. Here, $n$ is such that $\operatorname{Re} \lambda_{n}>0$.

$c_{n}=\sum_{\substack{i \\ \operatorname{Re} \lambda_{i}^{i}<0}} \sum_{\substack{j \\ \operatorname{Re} \lambda_{j}<0}} \alpha_{i j}^{n} \frac{c_{i} c_{j}}{\lambda_{i}+\lambda_{j}-\lambda_{n}}+\sum_{\substack{i \\ \operatorname{Re} \lambda_{i}<0}} \sum_{\substack{j \\ \operatorname{Re} \lambda_{j}<0}} \sum_{\substack{k \\ \operatorname{Re} \lambda_{k}<0}} \beta_{i j k}^{n} c_{i} c_{j} c_{k} \frac{1}{\lambda_{i}+\lambda_{j}+\lambda_{k}-\lambda_{n}}$

$-\sum_{\substack{i \\ \operatorname{Re} \lambda_{i}<0}} \sum_{\substack{j \\ \operatorname{Re} \lambda_{j}<0}} \sum_{\substack{k \\ \operatorname{Re} \lambda_{k}<0}} \sum_{\substack{l \\ \operatorname{Re} \lambda_{l}<0}}\left\{\alpha_{i j}^{n} \alpha_{k l}^{j}+\alpha_{j i}^{n} \alpha_{k l}^{j}\right\} c_{i} c_{k} c_{l} \frac{1}{\left(\lambda_{k}+\lambda_{l}+\lambda_{i}-\lambda_{n}\right)\left(\lambda_{i}+\lambda_{j}-\lambda_{n}\right)}$

$$
+\sum_{\substack{i \\ \operatorname{Re} \lambda_{i}<0}} \sum_{\substack{j \\ \operatorname{Re} \lambda_{j}>0}} \sum_{\substack{k \\ \operatorname{Re} \lambda_{k}<0}} \sum_{\substack{l \\ \operatorname{Re} \lambda_{l}<0}}\left\{\alpha_{i j}^{n} \alpha_{k l}^{j}+\alpha_{j i}^{n} \alpha_{k l}^{j}\right\} c_{i} c_{k} c_{l} \frac{1}{\left(\lambda_{k}+\lambda_{l}+\lambda_{i}-\lambda_{n}\right)\left(\lambda_{k}+\lambda_{l}-\lambda_{j}\right)} .
$$

This general formula can be applied, for example, to nonlinear elliptic problems of the form:

a) $\quad \nabla^{2} u=f(u, \mathbf{y}), \quad(x, \mathbf{y}) \in[\tau, \infty) \times \Omega$;

b) $\quad B_{\Omega} u=0, \quad \mathrm{y} \in \partial \Omega$;

c) $\quad \lim _{x \rightarrow \infty} u(x, \mathbf{y})=u_{\infty}(\mathbf{y})$

where $u_{\infty}(\mathbf{y})$ satisfies;
a) $\quad \nabla_{\mathbf{y}}^{2} u_{\infty}=f\left(u_{\infty}, \mathbf{y}\right), \quad \mathbf{y} \in \Omega$;
b) $\quad B_{\Omega} u_{\infty}=0, \quad \mathrm{y} \in \partial \Omega$.

The linearized equation in the tail is given by:

$$
\nabla^{2} v-f_{u}\left(u_{\infty}, \mathbf{y}\right) v=0
$$


which is of the form analyzed in $\S 3$. The condition that (6.15) have an exponential dichotomy is that all eigenvalues, $\alpha_{n}$, of the problem

a) $\quad \nabla_{\mathbf{y}}^{2} Y_{n}-f_{u}\left(u_{\infty}, \mathbf{y}\right) Y_{n}=\alpha_{n} Y_{n}, \quad \mathbf{y} \in \Omega$

b) $\quad B_{\Omega} Y_{n}=0, \quad \mathrm{y} \in \partial \Omega$;

be negative. Then, the following boundary condition can be derived from (6.12):

$$
\begin{aligned}
& c_{n}^{\prime}=-\lambda_{n} c_{n}-\sum_{i=1}^{\infty} \sum_{j=1}^{\infty} \alpha_{i j}^{n} \frac{c_{i} c_{j}}{\lambda_{i}+\lambda_{j}+\lambda_{n}}-\sum_{i=1}^{\infty} \sum_{j=1}^{\infty} \sum_{k=1}^{\infty} \beta_{i j k}^{n} \frac{c_{i} c_{j} c_{k}}{\lambda_{i}+\lambda_{j}+\lambda_{k}+\lambda_{n}} \\
&+\sum_{i=1}^{\infty} \sum_{j=1}^{\infty} \sum_{k=1}^{\infty} \sum_{l=1}^{\infty} \alpha_{i j}^{n} \alpha_{k l}^{j} \frac{c_{i} c_{k} c_{l}}{\lambda_{k}+\lambda_{l}+\lambda_{i}+\lambda_{n}}\left(\frac{1}{\lambda_{k}+\lambda_{l}+\lambda_{j}}-\frac{1}{\lambda_{i}+\lambda_{n}+\lambda_{j}}\right) \\
& n=1,2,3, \cdots .
\end{aligned}
$$

Here we have:

$$
\begin{aligned}
& c_{i}=\int_{\Omega} d \mathbf{y} Y_{i}(\mathbf{y}) V(\tau, \mathbf{y}) ; \\
& \lambda_{i}=\sqrt{-\alpha_{i}} \\
& \alpha_{i j}^{n}=\int_{\Omega} d \mathbf{y} \frac{1}{2} f_{u u}\left(u_{\infty}, \mathbf{y}\right) Y_{i}(\mathbf{y}) Y_{j}(\mathbf{y}) Y_{n}(\mathbf{y}) ; \\
& \beta_{i j k}^{n}=\int_{\Omega} d \mathbf{y} \frac{1}{6} f_{u u u}\left(u_{\infty}, \mathbf{y}\right) Y_{i}(\mathbf{y}) Y_{j}(\mathbf{y}) Y_{k}(\mathbf{y}) Y_{n}(\mathbf{y}) .
\end{aligned}
$$

The quadratic approximation to this condition is used in a numerical computation by the authors in [8].

\section{REFERENCES}

[1] S. Agmon and L. Nirenberg, Properties of solutions of ordinary differential equations in Banach space, Comm. Pure Appl. Math., 16 (1963), pp. 121-239.

[2] A. Bayliss, M. Gunzburger AND E. Turkel, Boundary conditions for the numerical solution of elliptic equations in exterior regions, SIAM J. Appl. Math., 42 (1982), pp. 430-451.

[3] J. BerEZANSKiI, Expansions in eigenfunctions of self-adjoint operators, Transl. Math. Mon. 17, American Mathematical Society, Providence, RI, 1968.

[4] J. DaletskiY AND M. KREIN, Stability of solutions of differential equations in Banach space, Transl. Math. Mon. 43, American Mathematical Society, Providence, RI, 1974.

[5] F. de Hoog AND R. WeIss, An approximation method for boundary value problems on infinite intervals, Computing, 24 (1980), pp. 227-239.

[6] B. GuSTAFSSON AND H.-O. KREISS, Boundary conditions for time dependent problems with an artificial boundary, J. Comp. Phys., 30 (1979), pp. 333-351.

[7] T. Hagstrom, Reduction of unbounded domains to bounded domains for partial differential equation problems, Ph. D. thesis, California Inst. of Technology, Pasadena, 1983.

[8] T. Hagstrom AND H. B. Keller, The numerical solution of semi-linear elliptic problems in unbounded cylindrical domains, to appear.

[9] - The numerical calculation of traveling wave solutions of parabolic equations, to appear.

[10] A. Jepson AND H. B. Keller, Asymptotic boundary conditions for ordinary differential equations, to appear.

[11] H. B. KELlER AND M. LENTINI, Boundary value problems over semi-infinite intervals and their numerical solution, SIAM J. Numer. Anal., 17 (1980), pp. 577-604.

[12] P. MARKowich $A$ theory for the approximation of solutions of boundary value problems on infinite intervals, this Journal, 13 (1982), pp. 484-513. 